\title{
Trigeminal neuropathic pain as a complication of anterior temporal lobectomy: report of 2 cases
}

\author{
Impreet Gill, BMSc, ${ }^{1}$ Andrew G. Parrent, MD, FRCSC, ${ }^{2}$ and David A. Steven, MD, MPH, FRCSC ${ }^{2}$ \\ ${ }^{1}$ Schulich School of Medicine and Dentistry and Faculty of Science; and 2Division of Neurosurgery, Department of Clinical \\ Neurological Sciences, Schulich School of Medicine and Dentistry, Western University, London, Ontario, Canada
}

\begin{abstract}
Cranial nerve (CN) deficits following anterior temporal lobectomy (ATL) are an uncommon but well-recognized complication. The usual CNs implicated in post-ATL complications include the oculomotor, trochlear, and facial nerves. To the authors' knowledge, injury to the trigeminal nerve leading to neuropathic pain has not been previously described in the literature. This paper presents 2 cases of trigeminal neuropathic pain following temporal lobe resections for pharmacoresistant epilepsy. The possible pathophysiological mechanisms are discussed and the microsurgical anatomy of surgically relevant structures is reviewed.
\end{abstract}

http://thejns.org/doi/abs/10.3171/2015.5.JNS15123

KEY WORDS anterior temporal lobectomy; epilepsy; trigeminal nerve; complications; pain

A NTERIOR temporal lobectomy (ATL) is a commonly performed, well-established, effective procedure for the treatment of pharmacoresistant temporal lobe epilepsy, with rates of seizure freedom following ATL ranging from $55 \%$ to $78 \%{ }^{5,6,16,18}$ Although it is a safe procedure, ATL is not without risks. The most commonly reported complications following ATL include infection, intracranial hematoma, hemiparesis, visual field deficits, and cranial nerve $(\mathrm{CN})$ deficits. ${ }^{10,15}$ Of the latter, the facial (CN VII), trochlear (CN IV), and oculomotor (CN III) nerves are most commonly injured. ${ }^{1,4,7,9,10}$ Diplopia, attributable to CN III and IV dysfunction, has been documented in as many as $15 \%$ of cases, $, 2,4,7,9$ while facial paralysis, derived from branches of $\mathrm{CN}$ VII, has been reported in as many as $3 \%$ of cases. ${ }^{1,10}$ In this paper we present 2 cases of trigeminal neuropathic pain that resulted as a complication of ATL. To our knowledge, this specific complication has not been previously reported.

\section{Case Reports}

Case 1

History and Examination

A 59-year-old right-handed man presented with a history of medically refractory dyscognitive seizures. His seizures began at the age of 45 years after probable viral encephalitis. The episodes were characterized by loss of awareness and staring followed by coughing and oral automatisms. The patient was amnestic for these episodes, which lasted less than a minute and were occurring at a 
frequency of 2 to 3 per week. MRI revealed bilateral hippocampal T2 hyperintensity without atrophy. Continuous scalp video electroencephalography (EEG) revealed bitemporal interictal spikes that were more frequent on the right. Recorded seizures all originated from the right anterior mesial temporal region. Due to the bitemporal interictal findings and bilateral MRI abnormalities, the patient underwent bitemporal subdural electrode monitoring with subdural strips. Our technique for temporal subdural strip electrode insertion has been previously described. ${ }^{14}$ The invasive recordings revealed a clear right mesial temporal onset.

\section{Operation}

Nine months after the invasive EEG, the patient underwent a right ATL using our previously described technique. ${ }^{8,18}$ Essentially, a standard ATL was performed using a subpial aspiration technique. The neocortical resection included the superior temporal gyrus and extended $5 \mathrm{~cm}$ from the tip of the temporal lobe. The mesial resection included the uncus, amygdala, and anterior hippocampus. The posterior extent of the mesial resection was at the level of the lateral mesencephalic sulcus. There were no intraoperative complications.

\section{Postoperative Course}

Immediately after surgery the patient complained of irritating numbness localized over the right side of the face, and was found to have decreased sensation to both light touch and pinprick in the distribution of all 3 divisions of the right trigeminal nerve. Six months after surgery the patient was seizure free, but the facial numbness persisted, especially in the V3 distribution. The numbness was associated with a mild, constant pain over the right side of the face with an occasional shooting pain in the right V1 distribution. Over time, this evolved into a constant rightsided facial pain, punctuated with occasional shocks of more intense pain. The pain was most severe in the V1 and V3 distributions, with associated pain and numbness in the teeth and tongue. The patient complained of facial sensitivity and ipsilateral sensitive teeth. One year following surgery, the patient reported new pain underneath the scalp flap that was found to be related to protruding cranial hardware. Surgical removal of this hardware eliminated the scalp discomfort, but did not help the facial pain. At 3 years postoperatively, the patient remained seizure free, but continued to have facial numbness and pain, although the latter was helped significantly with $1800 \mathrm{mg}$ /day of gabapentin.

\section{Case 2}

History and Examination

A 47-year-old right-handed man presented with a history of medically intractable dyscognitive seizures beginning at the age of 28 years. The episodes were characterized by the presence of a gargling sound with behavioral arrest and dystonic posturing of the left arm, followed by staring, unresponsiveness, and postictal confusion lasting 10-20 minutes. Interictal EEG revealed frequent spikes in the right temporal and frontal regions. MRI results were normal. Four complex partial seizures with simultaneous onset over the right mesial temporal and right frontal polar regions were also detected. Due to the lack of a clear onset on interictal EEG and normal MRI, the patient was investigated using subdural strip electrodes, which revealed a clear right anterior temporal onset.

\section{Operation}

Five months after the invasive EEG, the patient underwent a right ATL using the technique described above. There were no intraoperative complications.

\section{Postoperative Course}

Initially following the surgery the patient began complaining of severe pain over the right temporal bur hole site, where the subdural electrodes had been inserted 5 months previously. The pain was constant but could be exacerbated with even mild touch over that area. The cause of this pain was uncertain as the bur hole was posterior to the craniotomy used for the temporal lobectomy and was not exposed during the second procedure. The patient also complained of a second distinct type of pain involving the right side of the face. The patient described a shock-like sensation around his right eye. The pain was paroxysmal in nature and could be brought on while brushing his teeth or by wind. At 1 year after surgery the patient was free of seizures. The facial pain had disappeared entirely, but the bur hole pain persisted.

\section{Discussion}

Cranial nerve palsies are a documented complication following temporal lobectomy, with dysfunction of the oculomotor and trochlear nerves noted in as many as $15 \%$ of cases $^{2,4,7,9}$ and facial nerve palsies in as many as 3\% of cases. ${ }^{1,10}$ These extraaxial CN palsies have been attributed to both direct and indirect injury corresponding to their anatomy. After exiting the brainstem, the oculomotor nerve passes through the subarachnoid space immediately medial to the uncus of the temporal lobe. ${ }^{2,4}$ This nerve is often visible through the mesial leptomeninges and is usually situated a few millimeters away from the pial surface. In our experience, this nerve is rarely injured at surgery in the absence of adherence of the nerve to the mesial leptomeninges or in the setting of substantial uncal herniation such as would be present in the setting of a temporal lobe tumor. The trochlear nerve is rarely evident at surgery as it passes along the edge of the tentorium and it has been suggested that this lack of visibility renders it more prone to injury than the oculomotor nerve. ${ }^{4}$ Certainly, the position of the nerve at the tentorial edge renders it susceptible to direct and indirect trauma related to suction, cautery, ultrasonic aspiration, retraction, and other forms of surgical trauma that occur as the medial temporal structures are removed. Partial facial weakness after temporal lobectomy can be due to injury to the inferior Rolandic cortex or injury to the temporal branch of the facial nerve with an anteriorly placed preauricular limb of the skin incision. True complete facial nerve palsy after temporal lobectomy is harder to explain. Anderson et al. theorized that electrocoagulation of the dura overly- 
ing the geniculate ganglion might lead to a delayed complete facial paralysis. ${ }^{1}$

Injury to the trigeminal nerve during temporal lobe surgery has not been reported frequently. After exiting the pons, the motor and sensory roots of the trigeminal nerve course through the cerebellopontine angle cistern prior to exiting the posterior fossa via Meckel's cave, which is situated just below the superior petrosal sinus and tentorium cerebelli, and along the anterior surface of the petrous apex of the temporal bone., ${ }^{3,12,13}$ The superomedial wall of Meckel's cave associates closely with the lateral surface of the cavernous sinus, just below the oculomotor and trochlear nerves. ${ }^{13}$ Sabanc1 et al. noted that a wall separating Meckel's cave from the cavernous sinus could not be identified. ${ }^{13}$ A disturbance to the cavernous sinus may thus be transmitted directly to Meckel's cave. Exiting Meckel's cave, the ophthalmic and maxillary divisions of the trigeminal nerve course along the lateral wall of the cavernous sinus, ${ }^{11}$ while the mandibular division exits the cranium via the foramen ovale. It is in this location, along the lateral wall of the posterior cavernous sinus, or in the region of Meckel's cave, that the trigeminal nerve could theoretically be injured, presumably indirectly with thermal or electrocautery in the medial portion of the middle fossa.

Dysfunction of the trigeminal nerve following temporal lobectomy is unusual and, to our knowledge, has only been reported once in the literature. Thomas et al. reported a single case of transient unilateral xerophthalmia and sensory loss in the maxillary division (V2) of the trigeminal nerve following a hippocampal-sparing temporal resection for a cavernous malformation. ${ }^{17}$ The procedure was notable for requiring significant thermocoagulation and bipolar cautery to control bleeding on the floor of the middle fossa. With this case, tearing returned to normal and the facial numbness subsided over the course of 9 months following the operation. The authors theorized that the excessive thermocoagulation or bipolar cautery might have led to extradural injury to the ipsilateral trigeminal and greater superficial petrosal nerves. In neither of our 2 cases was an excessive need for coagulation noted, although this does not preclude the possibility that the trigeminal nerve was injured in such a manner. In the first case, facial numbness and pain were present immediately, which certainly implies that the nerve was injured during the procedure, presumably by the same mechanism. The onset of a symptom complex similar to trigeminal neuralgia in the second patient is more difficult to explain because there were no physical findings, such as facial numbness, that would implicate nerve injury. Although the patient had local pain around the previous bur hole, this incision was posterior to the ear and was not actually opened for the temporal lobectomy. Furthermore, the location of the "bur hole pain" was well within the C-2 dermatome and could not be neuroanatomically linked to the periorbital pain. The patient's facial pain was distinct from the other pain and very characteristic of classic trigeminal neuralgia. It also resolved completely. One possibility is that the brain shift or CSF drainage during surgery led indirectly to traction or microvascular compression of the trigeminal nerve in the cerebellopontine angle cistern. It is also possible that the pain was simply due to occult injury as was likely the case in the first patient. We considered anatomical variations as a potential contributing factor in both cases, but retrospective reviews of the MR images did not reveal any explanatory abnormalities related to the trigeminal nerve. In the end, we concluded that the most likely cause in both cases was an occult injury to the nerve due to cautery in the region of the mesial temporal lobe.

MRI-guided stereotactic laser thermal amygdalohippocampotomy is an emerging technique that is being used to treat mesial temporal lobe epilepsy. ${ }^{19}$ Although it is a closed procedure, it is theoretically possible that a similar complication could occur secondary to thermal injury to the trigeminal nerve. Practitioners performing all forms of open or closed surgery in the region of the mesial temporal lobe should be cognizant of the potential for thermal injury to the trigeminal nerve.

We encountered 2 unique and independent cases of trigeminal neuropathic pain following ATL for mesial temporal lobe epilepsy. One case presented with signs and symptoms consistent with an acute injury to the trigeminal nerve and the other presented as typical trigeminal neuralgia. Surgeons performing these procedures should be aware of this potential complication and take measures to avoid it. In particular, use of thermal and electrocautery in the medial portion of the middle fossa should be avoided.

\section{References}

1. Anderson J, Awad IA, Hahn JF: Delayed facial nerve palsy after temporal lobectomy for epilepsy: report of four cases and discussion of possible mechanisms. Neurosurgery 28:453-456, 1991

2. Bhatti MT, Eisenschenk S, Roper SN, Guy JR: Superior divisional third cranial nerve paresis: clinical and anatomical observations of 2 unique cases. Arch Neurol 63:771-776, 2006

3. Burr HS, Robinson GB: An anatomical study of the gasserian ganglion with particular reference to the nature and extend of Meckel's Cave. Anat Rec 29:269-282, 1925

4. Cohen-Gadol AA, Leavitt JA, Lynch JJ, Marsh WR, Cascino GD: Prospective analysis of diplopia after anterior temporal lobectomy for mesial temporal lobe sclerosis. J Neurosurg 99:496-499, 2003

5. Cohen-Gadol AA, Wilhelmi BG, Collignon F, White JB, Britton JW, Cambier DM, et al: Long-term outcome of epilepsy surgery among 399 patients with nonlesional seizure foci including mesial temporal lobe sclerosis. J Neurosurg 104:513-524, 2006

6. Engel J Jr, Van Ness PC, Rasmussen TB, Ojemann LM: Outcome with respect to epileptic seizures, in Engel J Jr (ed): Surgical Treatment of the Epilepsies, ed 2. New York: Raven Press, 1993, pp 609-621

7. Falconer MA, Serafetinides EA: A follow-up study of surgery in temporal lobe epilepsy. J Neurol Neurosurg Psychiatry 26:154-165, 1963

8. Girvin JP: Temporal lobectomy, in Apuzzo MLJ (ed): Neurosurgical Aspects of Epilepsy. Park Ridge, IL: American Association of Neurological Surgeons, 1991, pp 157-170

9. Jacobson DM, Warner JJ, Ruggles KH: Transient trochlear nerve palsy following anterior temporal lobectomy for epilepsy. Neurology 45:1465-1468, 1995

10. Pilcher WH, Roberts DW, Flanigin HF, Crandall PH, Wieser 
HG, Ojemann GA: Complications of epilepsy surgery, in Engel J Jr (ed): Surgical Treatment of the Epilepsies, ed 2. New York: Raven Press, 1993, pp 565-581

11. Rhoton AL Jr: The cavernous sinus, the cavernous venous plexus, and the carotid collar. Neurosurgery 51 (4 Suppl):S375-S410, 2002

12. Rhoton AL Jr: The temporal bone and transtemporal approaches. Neurosurgery 47 (3 Suppl):S211-S265, 2000

13. Sabancı PA, Batay F, Civelek E, Al Mefty O, Husain M, Abdulrauf SI, et al: Meckel's cave. World Neurosurg 76:335341, 266-267, 2011

14. Steven DA, Andrade-Souza YM, Burneo JG, McLachlan RS, Parrent AG: Insertion of subdural strip electrodes for the investigation of temporal lobe epilepsy. Technical note. J Neurosurg 106:1102-1106, 2007

15. Tanriverdi T, Ajlan A, Poulin N, Olivier A: Morbidity in epilepsy surgery: an experience based on 2449 epilepsy surgery procedures from a single institution. J Neurosurg 110:11111123,2009

16. Téllez-Zenteno JF, Dhar R, Wiebe S: Long-term seizure outcomes following epilepsy surgery: a systematic review and meta-analysis. Brain 128:1188-1198, 2005

17. Thomas AJ, Elisevich K, Smith B: Transient hemifacial sensory loss with xerophthalmia following temporal lobectomy. Can J Neurol Sci 27:251-253, 2000

18. Wiebe S, Blume WT, Girvin JP, Eliasziw M: A randomized, controlled trial of surgery for temporal-lobe epilepsy. $\mathbf{N ~ E n g l ~}$ J Med 345:311-318, 2001
19. Willie JT, Laxpati NG, Drane DL, Gowda A, Appin C, Hao $\mathrm{C}$, et al: Real-time magnetic resonance-guided stereotactic laser amygdalohippocampotomy for mesial temporal lobe epilepsy. Neurosurgery 74:569-585, 2014

\section{Disclosure}

The authors report no conflict of interest concerning the materials or methods used in this study or the findings specified in this paper.

\section{Author Contributions}

Conception and design: Steven. Acquisition of data: Steven. Analysis and interpretation of data: Steven, Gill. Drafting the article: Steven, Gill. Critically revising the article: all authors. Reviewed submitted version of manuscript: all authors. Approved the final version of the manuscript on behalf of all authors: Steven. Administrative/technical/material support: Steven, Gill. Study supervision: Steven.

\section{Correspondence}

David A. Steven, University Hospital, Rm. A10-323, 339

Windermere Rd., London, ON N6A5A5, Canada. email: david. steven@uwo.ca. 